\title{
QA Verification of Computer Codes Used in ORNL/TM-1999/159
}

\author{
P. T. Williams
}

B. R. Bass 


\title{
QA Verification of Computer Codes Used in ORNL/TM-1999/159
}

\author{
P. T. Williams \\ B. R. Bass \\ Computational Physics and Engineering Division
}

Manuscript Completed - February 2000

Date Published - March 2000

Prepared by the

OAK RIDGE NATIONAL LABORATORY

Oak Ridge, Tennessee 37831-8056

managed by

LOCKHEED MARTIN ENERGY RESEARCH CORP.

for the

U. S. DEPARTMENT OF ENERGY

under Contract No. DE-AC05-96OR22464 


\section{CONTENTS}

$\underline{\text { Page }}$

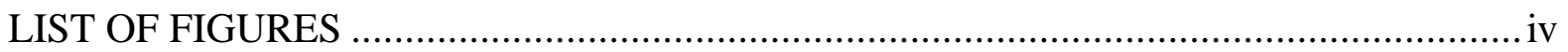

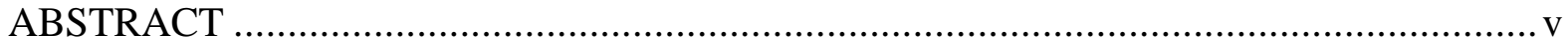

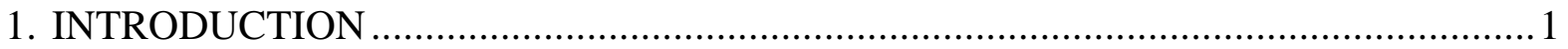

2. COMPUTER SYSTEMS AND CODES USED IN THE PREPARATION OF ORNL/TM-

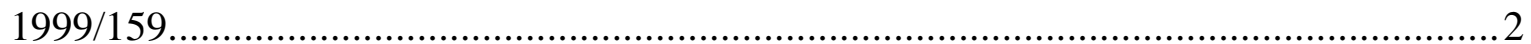

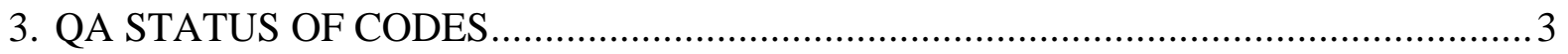

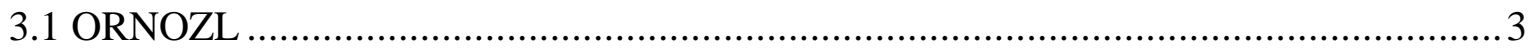

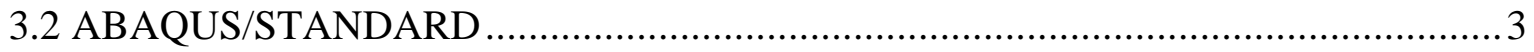

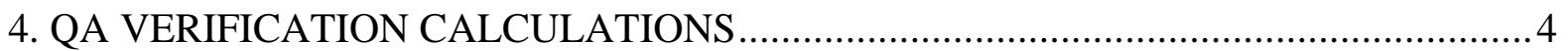

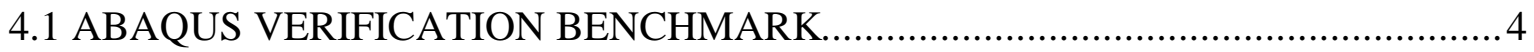

4.2 ORNOZL/ABAQUS VERIFICATION BENCHMARK..........................................

5. CONCLUSIONS

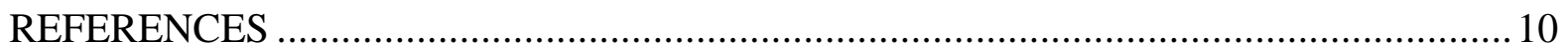

APPENDIX A: ORNOZL Output file (NOZZLE - I) Case Run: 11 February, 2000............. 11

APPENDIX B: ORNOZL Output file (NOZZLE - II) Case Run: 11 February, 2000............ 14

APPENDIX C:MATHCAD DATA SHEETS WITH EVALUATION OF APPROXIMATE STRESS-INTENSITY FACTORS USING THE GUZHONG AND QICHAO (1990) APPROXIMATION 


\section{LIST OF FIGURES}

Figure

$\underline{\text { Page }}$

Fig. 1. Listing of all files on the archival CD for ORNL/TM-1999/159......................2

Fig. 2. ORNOZL-generated finite-element models for (a) nozzle-I and (b) nozzle-II configurations using input data from Example Problems I and II in Ref. [4]..........3

Fig. 3. ABAQUS verification problem: linear-elastic stress-intensity factor for semielliptic surface flaw in a semi-infinite medium (a) Mode I tensile loading of surface flaw and (b) benchmark comparison with Newman-Raju solution.

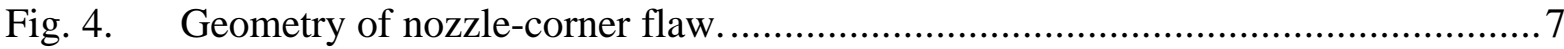

Fig. 5. Comparison of ABAQUS solutions to predictions of Guozhong and Qichao (1990). 


\begin{abstract}
This report describes QA verification exercises carried out for the computer codes applied in the analyses summarized "Stress Intensity Factors for HFIR HB-2 Nozzle Corner" (ORNL/TM-1999/159). Several benchmark problems are presented that establish the following: (1) The version of the finite-element mesh generator code ORNOZL used in the subject analyses reproduces the results of the two sample problems given in its previously published user's guide. (2) The ABAQUS code reproduces, independently of ORNOZL, the results of a benchmark verification problem given in its Example Problems Manual that compares linear-elastic stress intensity factors for semi-elliptical surface flaws to solutions published in the literature. (3) The ORNOZL/ABAQUS code combination was benchmarked against an approximate method for estimating linear-elastic stress-intensity factors for corner flaws in pressure vessel nozzles. In addition, all input and output files produced during the analyses described in ORNL/TM-1999/159 have been archived on an electronic medium (CD-R74-ORNL/TM-1999/159) and transmitted with this report to ORNL Research Reactors Division personnel for archival storage.
\end{abstract}




\section{INTRODUCTION}

In support of probabilistic fracture mechanics (PFM) studies for the High Flux Isotope Reactor (HFIR) Vessel Life Extension Program [1], a report entitled "Stress Intensity Factors for HFIR HB-2 Nozzle Corner," (ORNL/TM-1999/159) [2] was recently issued that presented the results of stress analyses of the HFIR HB-2 beam tube in which linear-elastic fracture mechanics (LEFM) stress intensity factors for postulated corner flaws were calculated. The study described in ORNL/TM-1999/159 employed two computer codes: (1) the ORNOZL mesh generation code and (2) the ABAQUS stress-analysis code. This report provides supporting documentation of QA verification for these two codes. 


\section{COMPUTER SYSTEMS AND CODES USED IN THE PREPARATION OF ORNL/TM-1999/159}

All finite-element stress analysis and $J$-integral calculations were carried out in the subject study using the ABAQUS/Standard, (version 5.7-1) [3] code on an IBM Risc/6000 Model 560 workstation computer running under AIX operating system 4.2.1.0. Model preparation required the use of the mesh-generation program ORNOZL [4] which was developed at ORNL to produce fully 3-dimensional finite-element models of nozzle-cylinder intersections containing a mathematically defined corner crack. Output from ORNOZL consists of files containing nodal point coordinates and element connectivities that completely define the geometry of the 3-dimensional model.

All input and output computer files used in the subject study have been stored on a compact disc, designated CD-R74-ORNL/TM-1999/159, and transmitted with this report to ORNL Research Reactors Division (RRD) personnel for archival storage. Additionally included on this CD are the FORTRAN source and IBM/AIX executable for the ORNOZL code. Since the ABAQUS/Standard computer code is a commercial program available from Hibbitt, Karlsson \& Sorensen, Inc., under network licensing, (i.e., ORNL does not own the source code or executable), the ABAQUS source code and executables are not available for archival storage. A listing of all files located on the archival CD is given in Fig. 1.

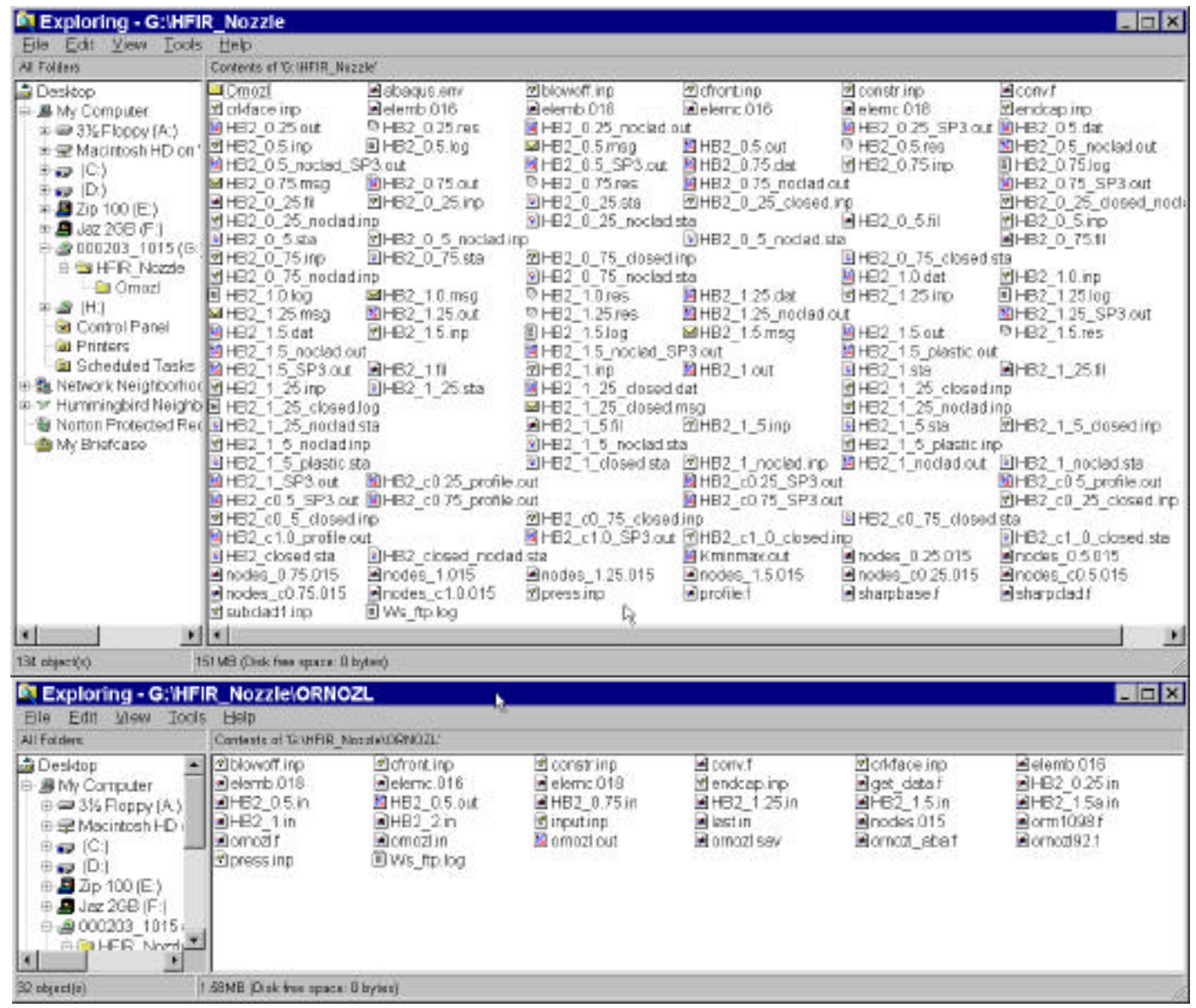

Fig. 1. Listing of all files on the archival CD for ORNL/TM-1999/159. 


\section{QA STATUS OF CODES}

\subsection{ORNOZL}

The ORNOZL finite-element mesh generation code was developed at ORNL in support of the US Nuclear Regulatory Commission (USNRC) sponsored Heavy Section Steel Technology (HSST) Program. The code's user's guide was documented in a USNCR NUREG report [4] which underwent internal ORNL peer review and USNRC review. The two test cases reported in [4] were rerun for this study and checked against the output listings given in ref. [4] (see Fig. 2 and Appendices A and B). The results of this check established that the current copy of ORNOZL reproduces the same output file as the code documented in 1992. The results of additional QA verification calculations are presented in Sect. 4.

\subsection{ABAQUS/STANDARD}

The developers of ABAQUS, (Hibbit, Karlson, and Sorenson, Inc., (HKS)) have implemented a Quality Assurance Plan that is based on the ANSI/ASME NQA-1 Quality Assurance standard, which is designed to ensure compliance with Appendix B of US federal regulation 10 CFR 50 (1-1-86), Quality Assurance Criteria for Nuclear Power Plants and Fuel Reprocessing Plants. HKS contracts annually with an independent quality assurance audit organization to audit HKS's quality assurance procedures. The audit organization is chosen for its experience and its ability to ensure that HKS complies with the provisions of the ISO 9001:1994 and NQA-1 quality standards. ABAQUS is certified under ISO 9001 Certificate No. A3897 issued by the Underwriters Laboratory, Inc. ${ }^{\circledR}$ (issue date: April 9, 1996; revision date: March 9, 1998; renewal date: April 9, 2000).

(a)

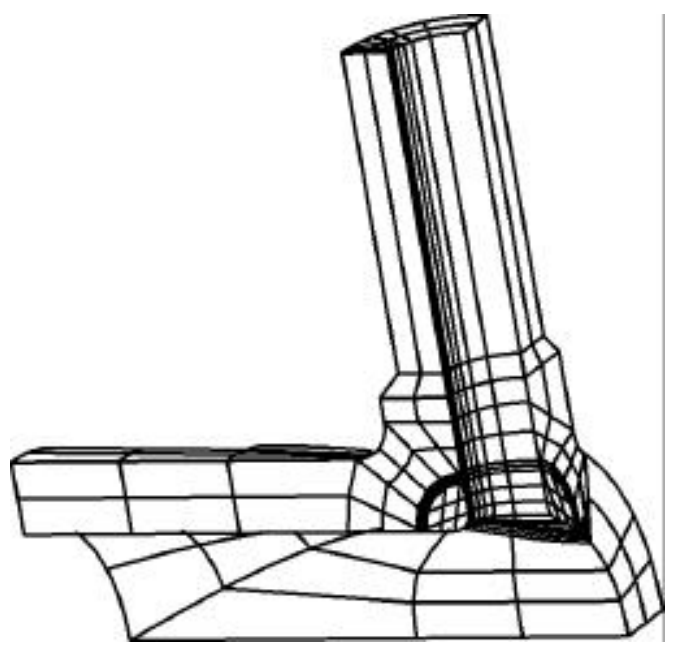

(b)

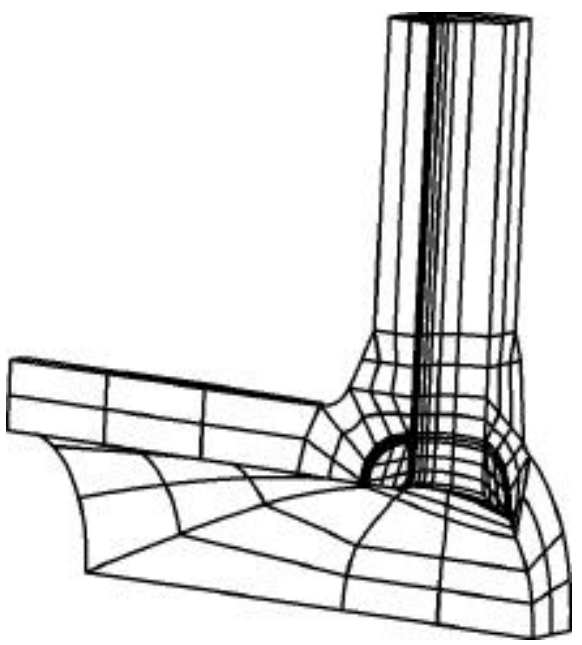

Fig. 2. ORNOZL-generated finite-element models for (a) nozzle-I and (b) nozzle-II configurations using input data from Example Problems I and II in Ref. [4] 


\section{QA VERIFICATION CALCULATIONS}

\subsection{ABAQUS Verification Benchmark}

Example Problem 3.1.7 in ref. [5] presents, as a verification benchmark, a comparison of stress intensity factors calculated from ABAQUS-generated $J$-integral results with linearelastic stress-intensity factors using the correlations developed by Newman and Raju in ref. [6] for a semi-elliptic surface flaw in a semi-infinite medium under a Mode I tensile loading (see Fig. 3). The $J$-integrals calculated by ABAQUS are converted into stressintensity factors, $K_{J}$, using the following plane-strain relation

$$
K_{J}=+\sqrt{\left(\frac{E}{1-v^{2}}\right) J}
$$

The $J$-integrals generated with the current check calculation are compared in Table 1 to the $J$-integral estimates published in ref. [5]. The comparison shows that the current study using ABAQUS reproduces the ABAQUS $J$-integral results reported in ref. [5], and there is good agreement between ABAQUS and the Newman-Raju [6] solution with the discrepancies in the results increasing as the flaw front approaches the free surface at $\phi=0$. As discussed in [5], the accuracy loss near the free surface is assumed to be attributable to the coarse and rather distorted mesh in this region.

(a)

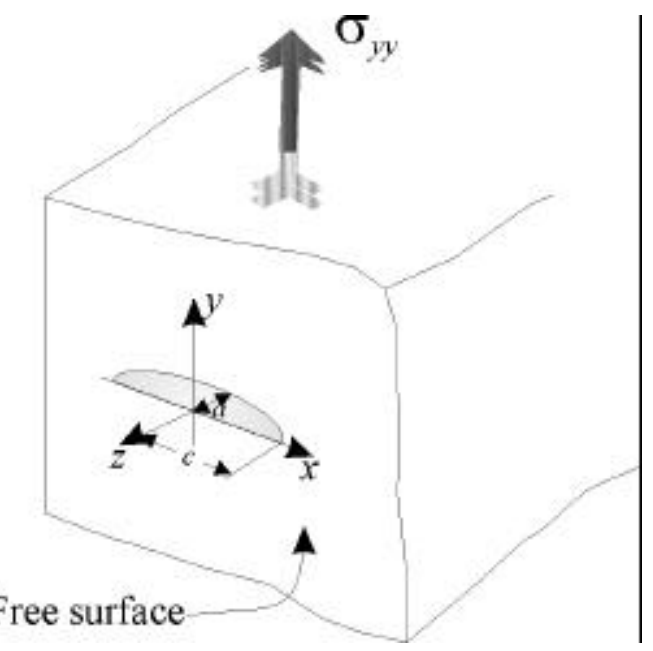

(b)

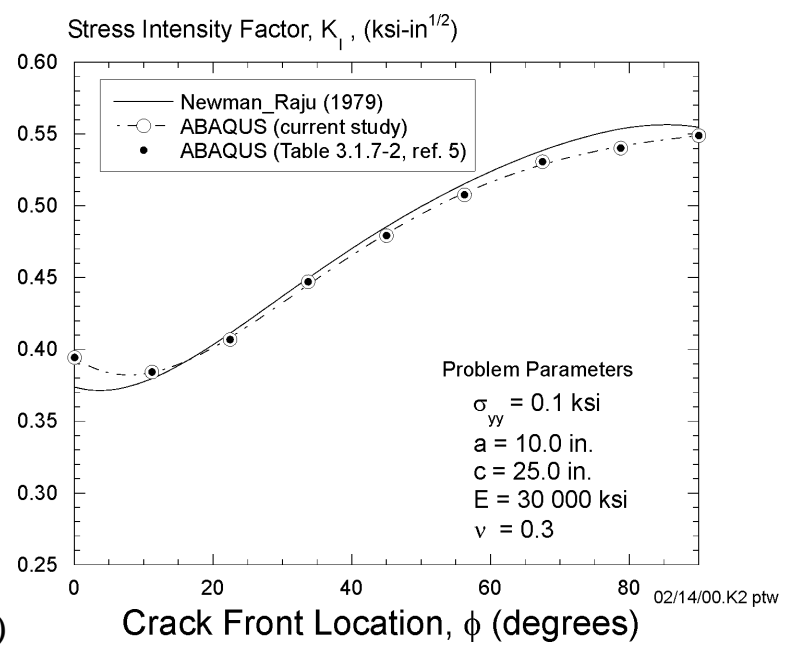

Fig. 3. ABAQUS verification problem: linear-elastic stress-intensity factor for semielliptic surface flaw in a semi-infinite medium (a) Mode I tensile loading of surface flaw and (b) benchmark comparison with Newman-Raju solution. 
Table 1. Comparison of Current ABAQUS Solution to Table 3.1.7-1 in Ref. [5]

\begin{tabular}{|c|c|c|c|c|c|c|c|c|}
\hline \multirow{3}{*}{$\begin{array}{c}\text { Crack Front } \\
\text { Location } \\
\text { (degrees) }\end{array}$} & \multicolumn{4}{|c|}{ Current ABAQUS $J$-integral estimates (lbf-in./in. ${ }^{2}$ ) } & \multicolumn{4}{|c|}{$J$-integral estimates from Table 3.1.7-1 in [5] (lbf-in./in. ${ }^{2}$ ) } \\
\hline & Contour & Contour & Contour & Average & Contour & Contour & Contour & Average \\
\hline & 1 & 2 & 3 & Value & 1 & 2 & 3 & Value \\
\hline 0.00 & 0.0047447 & 0.0046331 & 0.0047847 & 0.0047208 & 0.0047447 & 0.0046331 & 0.0047847 & 0.0047208 \\
\hline 11.25 & 0.0043670 & 0.0045162 & 0.0045531 & 0.0044788 & 0.0043670 & 0.0045162 & 0.0045531 & 0.0044787 \\
\hline 22.50 & 0.0051080 & 0.0050077 & 0.0049506 & 0.0050221 & 0.0051080 & 0.0050077 & 0.0049506 & 0.0050223 \\
\hline 33.75 & 0.0060384 & 0.0060625 & 0.0060953 & 0.0060654 & 0.0060384 & 0.0060625 & 0.0060953 & 0.0060656 \\
\hline 45.00 & 0.0069947 & 0.0069676 & 0.0069397 & 0.0069673 & 0.0069947 & 0.0069676 & 0.0069397 & 0.0069673 \\
\hline 56.25 & 0.0078177 & 0.0078212 & 0.0078161 & 0.0078183 & 0.0078177 & 0.0078212 & 0.0078161 & 0.0078183 \\
\hline 67.50 & 0.0085532 & 0.0085439 & 0.0085435 & 0.0085469 & 0.0085532 & 0.0085439 & 0.0085435 & 0.0085467 \\
\hline 78.75 & 0.0088540 & 0.0088523 & 0.0088484 & 0.0088516 & 0.0088540 & 0.0088523 & 0.0088484 & 0.0088516 \\
\hline 90.00 & 0.0091341 & 0.0091367 & 0.0091483 & 0.0091397 & 0.0091341 & 0.0091367 & 0.0091483 & 0.0091397 \\
\hline
\end{tabular}




\subsection{ORNOZL/ABAQUS Verification Benchmark}

The combination of the ORNOZL/ABAQUS codes used to produce finite-element solutions of stress-intensity factors for nozzle corner flaws has been benchmarked against an approximate method presented by Guozhong and Qichao [7]. In ref. [7], the iso-stress lines at a nozzle corner are simplified into slanted straight lines at an angle of $45^{\circ}$ with the walls of a cylindrical pressure vessel and nozzle. In this approximation, nozzle corner cracks are represented by simplified quarter-circular flaws. The vessel geometry, nozzle geometry, and associated nomenclature are depicted in Fig. 4. On the basis of the assumed stress profiles, $K_{I}$ solutions for an arbitrary point on the crack front were derived in [7] by an approximate analysis producing the following relation for a Mode I stress-intensity factor:

$$
K_{I}(\bar{a}, \theta)=M_{f}(\theta) M_{b}(\theta) k_{t}(\pi \bar{a} / 4, \theta) \frac{\sigma \sqrt{\pi \bar{a}}}{\pi / 2}
$$

where $M_{f}$ and $M_{b}$ are front and back free-surface magnification factors, respectively, assumed to have the forms

$$
\begin{aligned}
M_{f}(\theta) & =1.43-0.24(\sin \theta+\cos \theta) \\
M_{b}(\theta) & =1.0
\end{aligned}
$$

The parameter $k_{t}(r, \theta)$ is the local elastic stress concentration factor at a location $(r, \theta)$ from the origin (see Fig. 4), given by

$$
\begin{gathered}
k_{t}(r, \theta)=1+\left(k_{e}-1\right)\left(\frac{1}{1+(\sin \theta+\cos \theta)\left(r / r_{i}\right)}\right)^{B} \\
B=2.70-2 \sqrt{t / d}
\end{gathered}
$$

where $k_{e}$ is the elastic stress concentration factor at the origin which can be estimated by the Decock relation [8]

$$
k_{e}=\frac{2+2(d / D) \sqrt{(d t) /(D T)}+1.25(d / D)(D / T)}{1+(t / T) \sqrt{(d t) /(D T)}}
$$

where $d$ and $D$ are the mean diameters of the nozzle and vessel, respectively. A complete listing of the nomenclature used in Eqs. (2)-(6) is given in Appendix C.

The Guozhong and Qichao approximation [7] does not include the effects of cladding; therefore, finite-element solutions from the no-cladding cases in ORNL/TM-1999/159 were used for comparison. Figure 5a plots the maximum predicted $K_{I}$ solutions as a function of flaw radius for both the ORNOZL/ABAQUS linear-elastic solutions (taken from [2] for the nocladding cases) and the results of the approximate analysis of Guozhong and Qichao [7] for the HFIR HB-2 nozzle corner geometry (see Appendix C). As indicated by Fig. 5b, the maximum deviation between the two solution sets was $7.7 \%$ for the smallest flaw size investigated with the ORNOZL/ABAQUS solution being higher than the Guozhong and Qichao estimate. These deviations fall within the $\pm 10 \%$ uncertainty band established in ref. [7] using comparisons with published solutions for approximately 40 cracks from nine nozzle section geometries. 


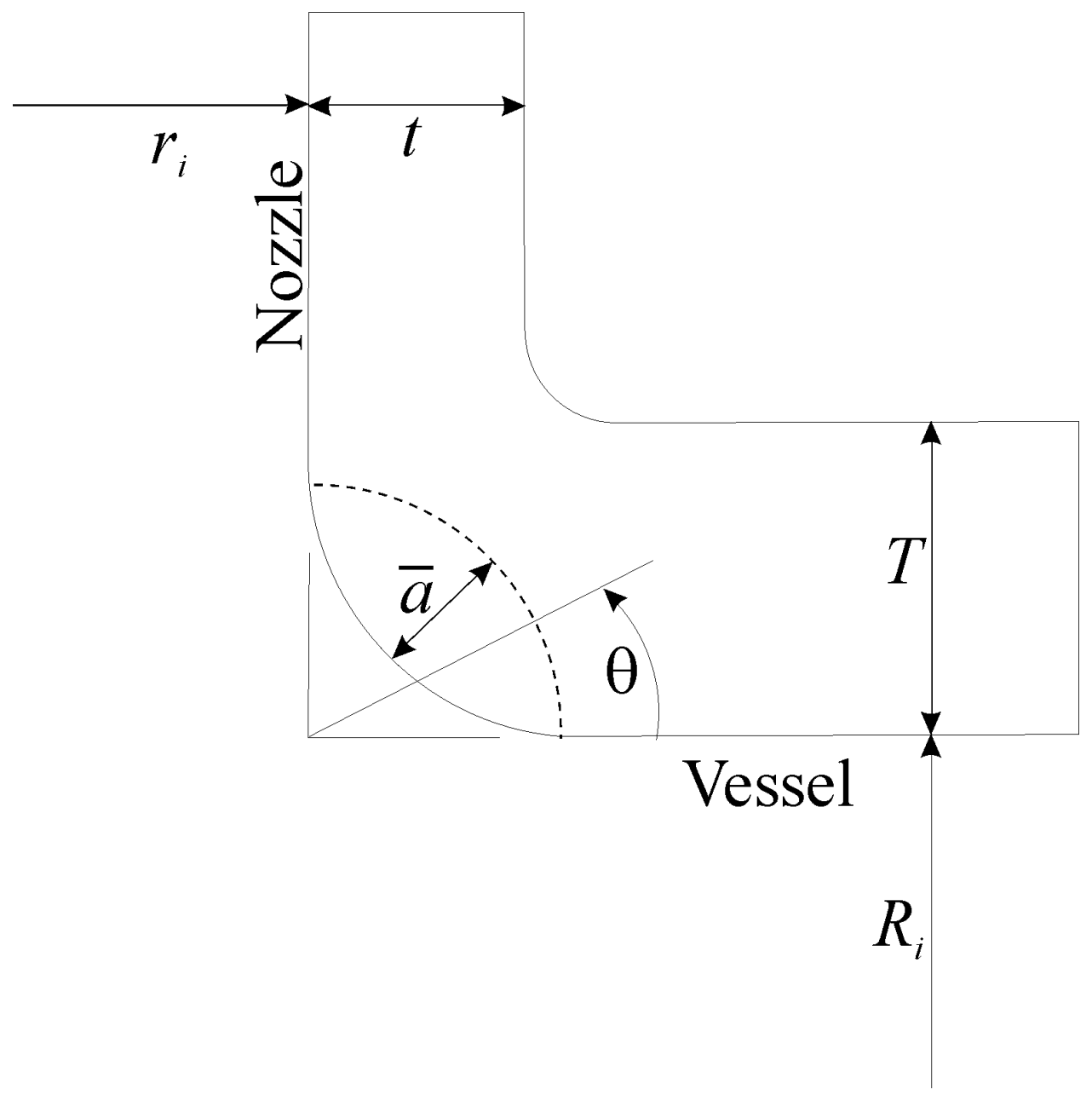

Fig. 4. Geometry of nozzle-corner flaw. 


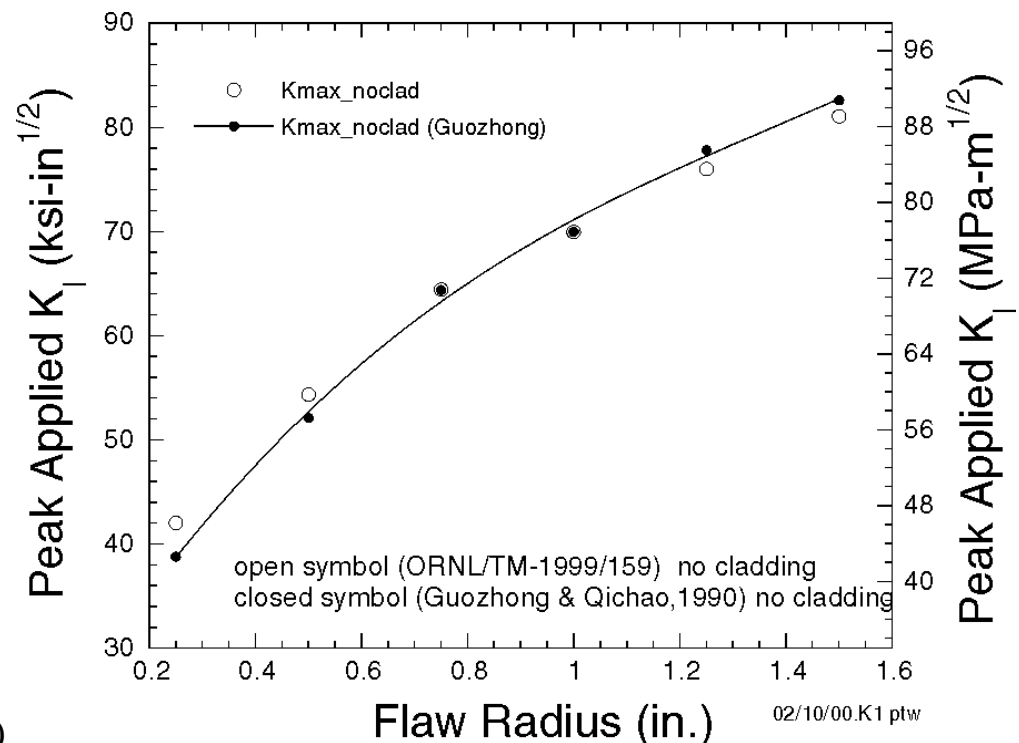

(a)

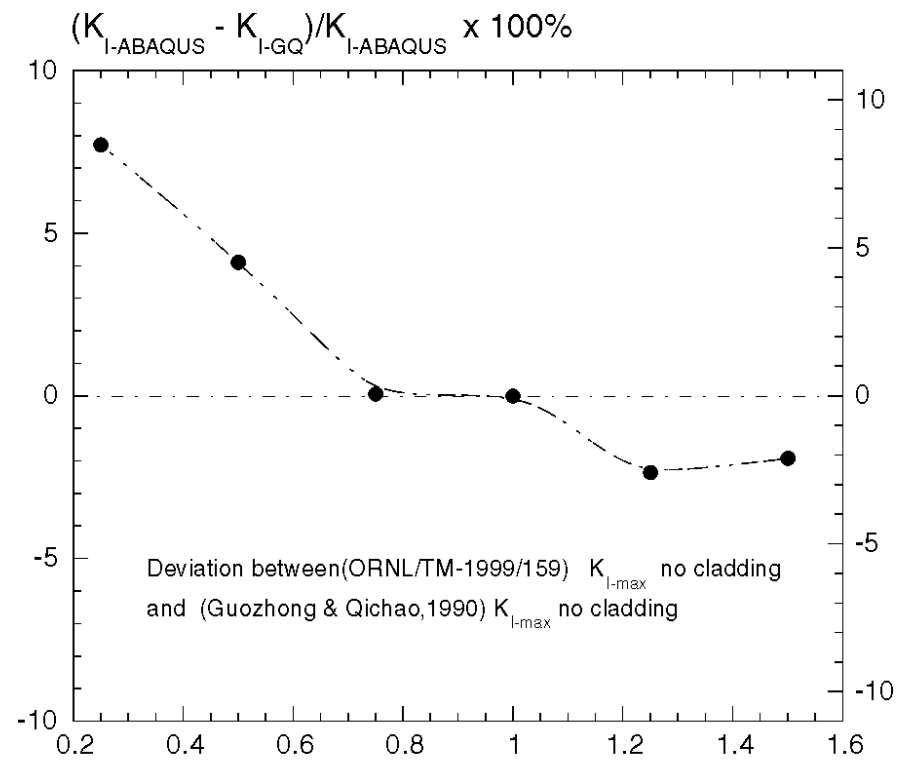

(b)

Flaw Radius (in.)

Fig. 5. Comparison of ABAQUS solutions to predictions of Guozhong and Qichao (1990). 


\section{CONCLUSIONS}

As a QA verification of the computer codes used in the report "Stress Intensity Factors for HFIR HB-2 Nozzle Corner" (ORNL/TM-1999/159) [2], benchmark problems have been solved that have established the following:

- The current version of the finite-element mesh generator code ORNOZL reproduces the results of the two sample problems given in its previously published user's guide [4].

- The ABAQUS code reproduces, independently of ORNOZL, the results of a benchmark verification problem given in its Example Problems Manual that compares linear-elastic stress intensity factors for semi-elliptic surface flaws to solutions published in the literature $[5,6]$.

- The ORNOZL/ABAQUS code combination was benchmarked against an approximate method [7] for estimating linear-elastic stress-intensity factors for corner flaws in pressure vessel nozzles.

- In addition, all input and output files produced during the analyses described in ORNL/TM-1999/159 have been archived on an electronic medium (CD-R74-ORNL/TM1999/159) and transmitted to RRD personnel for archival storage. 


\section{REFERENCES}

1. R. D. Cheverton and T. L. Dickson, HFIR Vessel Life Extension with Enlarged HB-2 and HB-4 Beam Tubes, ORNL/TM-13698, Lockheed Martin Energy Research, Oak Ridge Natl. Lab., December 1998.

2. P. T. Williams and B. R. Bass, "Stress Intensity Factors for HFIR HB-2 Nozzle Corner,” ORNL/TM-1999/159, Oak Ridge National Laboratory, August 1999.

3. ABAQUS Theory Manual, Version 5.7, Hibbit, Karlson, and Sorenson, Inc., Providence, RI, 1997.

4. J. Keeney-Walker and B. R. Bass, ORNOZL: A Finite-Element Mesh Generator for Nozzle-Cylinder Intersections Containing Inner-Corner Cracks, NUREG/CR-5872 (ORNL/TM-11049), Martin Marietta Energy Systems, Inc., Oak Ridge Natl. Lab., September 1992.

5. ABAQUS/Standard Example Problems Manual: Volume I, Hibbit, Karlson, and Sorenson, Inc., Providence, RI, (1997) pp. 3.1.7-1 to 3.1.7-18.

6. J. C. Newman and I. S. Raju, "Stress-Intensity Factors for a Wide Range of SemiElliptical Surface Cracks in Finite Thickness Plates," Engineering Fracture

7. C. Guozhong and H. Qichao, "Approximate Stress-Intensity Factor Solutions for Nozzle Corner Cracks," International Journal of Pressure Vessels and Piping 42, (1990) 75-96.

8. J. Decock, "Determination of Stress Concentrations Factors and Fatigue Assessment of Flush and Extruded Nozzles in Welded Pressure Vessels," $2^{\text {nd }}$ International Conference on Pressure Vessel Tech., Part II, ASME, San Antonio, Texas, Paper II-59, (1973) 821-834. 


\title{
APPENDIX A: ORNOZL OUTPUT FILE (NOZZLE - I) CASE RUN: 11 FEBRUARY, 2000
}

\author{
ornozl - 3 - d mes $h$ generator \\ ornl - hs st program \\ d e c e mber 1,1984
}

NOZZLE TEST CASE 1

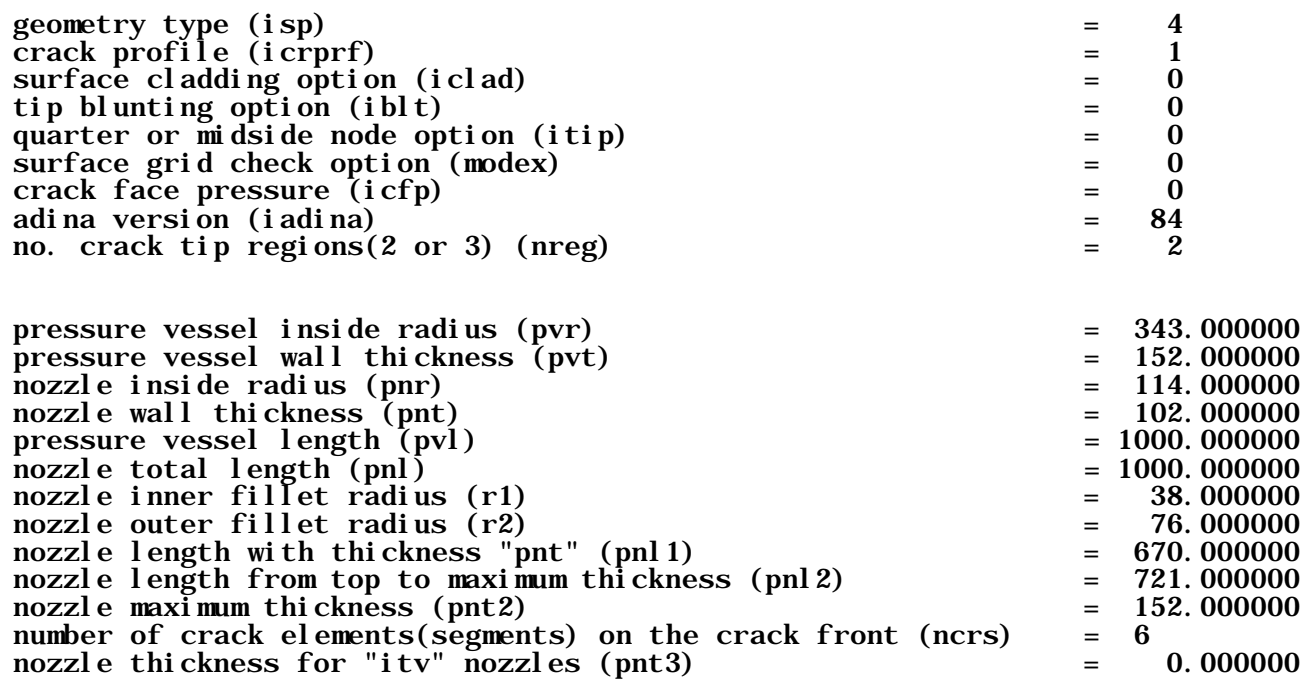$$
\begin{array}{rr}
= & 4 \\
= & 1 \\
= & 0 \\
= & 0 \\
= & 0 \\
= & 0 \\
= & 0 \\
= & 84 \\
= & 2
\end{array}
$$

$=0.000000$

fini te el ement grid breakdown :

$\begin{array}{lll}\mathrm{nx} & = & 2 \\ \mathrm{n} \times 2 & = & 3 \\ \mathrm{nx3} & = & 2 \\ \mathrm{nx4} & = & 1 \\ \mathrm{ny1} & = & 2 \\ \mathrm{ny2} & = & 2 \\ \mathrm{nz} & = & 6 \\ \text { si ze } & = & 5.00000 \\ \text { gradx } & = & 1.00000\end{array}$

crack defi ni ti on parameters :

$x$ di rection $(\mathrm{cra})=110.000000$

$y$ di rection $(\mathrm{crb})=110.000000$

the computed stress intensity factors would corresspond to the foll owi ng el I i ptical angl es

$\begin{array}{cccc}\begin{array}{c}\text { el ement } \\ \text { number }\end{array} & \begin{array}{c}\text { thet a } \\ \text { (radi ans) }\end{array} & \begin{array}{c}\text { thet a } \\ \text { (degr ees) }\end{array} & \text { thet a/ ( pi / 2) } \\ 1 & 0.302 \mathrm{E}-01 & 0.173 \mathrm{E}+01 & 0.192 \mathrm{E}-01 \\ 2 & 0.242 \mathrm{E}+00 & 0.138 \mathrm{E}+02 & 0.154 \mathrm{E}+00 \\ 3 & 0.604 \mathrm{E}+00 & 0.346 \mathrm{E}+02 & 0.385 \mathrm{E}+00 \\ 4 & 0.967 \mathrm{E}+00 & 0.554 \mathrm{E}+02 & 0.615 \mathrm{E}+00 \\ 5 & 0.133 \mathrm{E}+01 & 0.762 \mathrm{E}+02 & 0.846 \mathrm{E}+00 \\ 6 & 0.154 \mathrm{E}+01 & 0.883 \mathrm{E}+02 & 0.981 \mathrm{E}+00\end{array}$

nozzl e corner mesh parameters(generated): 


$\begin{array}{lll}\text { case no. ( } \mathrm{i} \mathrm{ndx} 1) & = \\ \mathrm{r} 1 & = & 0.38000 \mathrm{E}+02 \\ \text { xsht } & = & 0.10000 \mathrm{E}+03 \\ \text { ysht } & = & 0.10000 \mathrm{E}+03\end{array}$

the computed stress intensity factors would corresspond to the following elliptical angles

$\begin{array}{cccccc}\begin{array}{c}\text { node } \\ \text { number }\end{array} & \begin{array}{c}\text { thet } \mathrm{r} \text { (radi ans) } \\ \text { thet }\end{array} & \begin{array}{c}\text { thet } \mathrm{a} /(\mathrm{pi} / 2) \\ \text { (degr ees) }\end{array} & & & \\ 1 & 0.000 \mathrm{E}+00 & 0.000 \mathrm{E}+00 & 0.000 \mathrm{E}+00 & 0.900 \mathrm{E}+02 & 0.707 \mathrm{E}+00 \\ 2 & 0.302 \mathrm{E}-01 & 0.173 \mathrm{E}+01 & 0.192 \mathrm{E}-01 & 0.883 \mathrm{E}+02 & 0.708 \mathrm{E}+00 \\ 3 & 0.604 \mathrm{E}-01 & 0.346 \mathrm{E}+01 & 0.385 \mathrm{E}-01 & 0.865 \mathrm{E}+02 & 0.709 \mathrm{E}+00 \\ 4 & 0.242 \mathrm{E}+00 & 0.138 \mathrm{E}+02 & 0.154 \mathrm{E}+00 & 0.762 \mathrm{E}+02 & 0.736 \mathrm{E}+00 \\ 5 & 0.423 \mathrm{E}+00 & 0.242 \mathrm{E}+02 & 0.269 \mathrm{E}+00 & 0.658 \mathrm{E}+02 & 0.783 \mathrm{E}+00 \\ 6 & 0.604 \mathrm{E}+00 & 0.346 \mathrm{E}+02 & 0.385 \mathrm{E}+00 & 0.554 \mathrm{E}+02 & 0.838 \mathrm{E}+00 \\ 7 & 0.785 \mathrm{E}+00 & 0.450 \mathrm{E}+02 & 0.500 \mathrm{E}+00 & 0.450 \mathrm{E}+02 & 0.889 \mathrm{E}+00 \\ 8 & 0.967 \mathrm{E}+00 & 0.554 \mathrm{E}+02 & 0.615 \mathrm{E}+00 & 0.346 \mathrm{E}+02 & 0.933 \mathrm{E}+00 \\ 9 & 0.115 \mathrm{E}+01 & 0.658 \mathrm{E}+02 & 0.731 \mathrm{E}+00 & 0.242 \mathrm{E}+02 & 0.967 \mathrm{E}+00 \\ 10 & 0.133 \mathrm{E}+01 & 0.762 \mathrm{E}+02 & 0.846 \mathrm{E}+00 & 0.138 \mathrm{E}+02 & 0.989 \mathrm{E}+00 \\ 11 & 0.151 \mathrm{E}+01 & 0.865 \mathrm{E}+02 & 0.962 \mathrm{E}+00 & 0.346 \mathrm{E}+01 & 0.999 \mathrm{E}+00 \\ 12 & 0.154 \mathrm{E}+01 & 0.883 \mathrm{E}+02 & 0.981 \mathrm{E}+00 & 0.173 \mathrm{E}+01 & 0.100 \mathrm{E}+01 \\ 13 & 0.157 \mathrm{E}+01 & 0.900 \mathrm{E}+02 & 0.100 \mathrm{E}+01 & 0.000 \mathrm{E}+00 & 0.100 \mathrm{E}+01\end{array}$

angul ar di vi si on - nozzl e

$\begin{array}{rr}\text { di v } & \text { angl e } \\ 1 & 0.00000 \\ 2 & 1.25689 \\ 3 & 2.51378 \\ 4 & 3.77067 \\ 5 & 5.02756 \\ 6 & 7.54134 \\ 7 & 10.05512 \\ 8 & 14.17148 \\ 9 & 18.28785 \\ 10 & 22.04923 \\ 11 & 25.81061 \\ 12 & 30.88520 \\ 13 & 35.95980 \\ 14 & 45.69245 \\ 15 & 55.42510 \\ 16 & 72.71255 \\ 17 & 90.00000\end{array}$

angul ar di vi si on - vessel

$\begin{array}{rr}\text { di v } & \text { angl e } \\ 1 & 0.83525 \\ 2 & 15.69604 \\ 3 & 30.55683 \\ 4 & 45.41762 \\ 5 & 60.27842 \\ 6 & 75.13921 \\ 7 & 90.00000\end{array}$

mesh generati on parameters - subrouti ne revol v :

$\begin{array}{llr}\text { i n } & = & 3 \\ \text { i v } & = & 3 \\ \text { i } & = & 5 \\ \text { i } & = & 9 \\ \text { iff } & = & 11 \\ \text { i st } & = & 5 \\ \text { i ndx1 } & = & 2 \\ \text { nx } & = & 25 \\ \text { ny } & = & 13 \\ \text { nz1 } & = & 3 \\ \text { nz2 } & = & 3 \\ \text { nz3 } & = & 0 \\ \text { nozshp } & = & 2\end{array}$


pert urbati on vect or for crack front nodes

$\begin{array}{rrrr}\text { node } & \mathrm{d} 1 & \mathrm{~d} 2 & \mathrm{~d} 3 \\ 1 & 0.100000 \mathrm{E}+01 & 0.000000 \mathrm{E}+00 & 0.000000 \mathrm{E}+00 \\ 2 & 0.100000 \mathrm{E}+01 & 0.000000 \mathrm{E}+00 & 0.000000 \mathrm{E}+00 \\ 3 & 0.100000 \mathrm{E}+01 & 0.000000 \mathrm{E}+00 & 0.000000 \mathrm{E}+00 \\ 4 & 0.977101 \mathrm{E}+00 & 0.212778 \mathrm{E}+00 & 0.00000 \mathrm{E}+00 \\ 5 & 0.910891 \mathrm{E}+00 & 0.412647 \mathrm{E}+00 & 0.000000 \mathrm{E}+00 \\ 6 & 0.822262 \mathrm{E}+00 & 0.569109 \mathrm{E}+00 & 0.000000 \mathrm{E}+00 \\ 7 & 0.707107 \mathrm{E}+00 & 0.707107 \mathrm{E}+00 & 0.000000 \mathrm{E}+00 \\ 8 & 0.569106 \mathrm{E}+00 & 0.822264 \mathrm{E}+00 & 0.000000 \mathrm{E}+00 \\ 9 & 0.412650 \mathrm{E}+00 & 0.910890 \mathrm{E}+00 & 0.000000 \mathrm{E}+00 \\ 10 & 0.212774 \mathrm{E}+00 & 0.977101 \mathrm{E}+00 & 0.000000 \mathrm{E}+00 \\ 11 & 0.000000 \mathrm{E}+00 & 0.100000 \mathrm{E}+01 & 0.000000 \mathrm{E}+00 \\ 12 & 0.000000 \mathrm{E}+00 & 0.100000 \mathrm{E}+01 & 0.000000 \mathrm{E}+00 \\ 13 & 0.000000 \mathrm{E}+00 & 0.100000 \mathrm{E}+01 & 0.000000 \mathrm{E}+00\end{array}$

number of nodes i $n$ adi na crack model. . . . . $($ nod $)=2616$

number of crack tip el ements. . . . . . $($ nel t $)=96$

first el ement on crack front. . . . . . . $($ nre2 $)=49$

first el ement of out er regi on ...... . $($ nre3 $)=0$

number of regul ar el ement s. . . . . . $\quad($ nel $)=408$

number of i nternal pres el ements... . . (nprel $)=218$

number of crack face pres el ements . . . . (nprcf $)=0$ 


\title{
APPENDIX B: ORNOZL OUTPUT FILE (NOZZLE - II) CASE RUN: 11 FEBRUARY, 2000
}

\author{
ornozl- -3 - d mes $h$ ge $n$ e $r$ a tor \\ ornl - hsst program \\ d e c e mber $1, \quad 1984$
}

NOZZLE TEST CASE 1

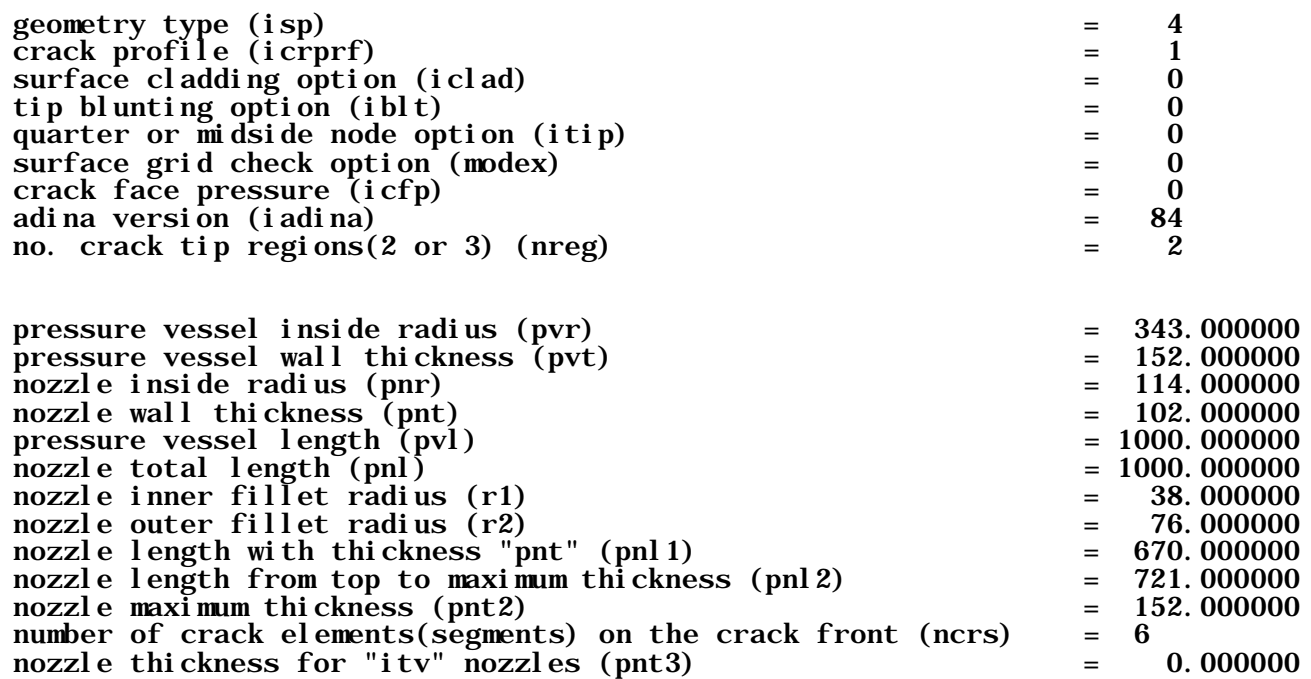

$\begin{array}{rr}= & 4 \\ = & 1 \\ = & 0 \\ = & 0 \\ = & 0 \\ = & 0 \\ = & 0 \\ = & 84 \\ = & 2\end{array}$

$=343.000000$
$=152.000000$

$=114.000000$

$=102.000000$

$=1000.000000$

$=1000.000000$

$=38.000000$

$=76.000000$

$=670.000000$

$=721.000000$

$=152.000000$

$=6$

$=0.000000$

fi ni te el ement grid breakdown :

$\begin{array}{lll}\text { nx1 } & = & 2 \\ \text { nx2 } & = & 3 \\ \text { nx3 } & = & 2 \\ \text { nx4 } & = & 1 \\ \text { ny1 } & = & 2 \\ \text { ny2 } & = & 6 \\ \text { nz } & = & 6.00000 \\ \text { size } & = & 1.00000\end{array}$

crack defi ni ti on parameters :

$x$ di rection $(\mathrm{cra})=110.000000$

$y$ di rection $(\mathrm{crb})=110.000000$

the computed stress i ntensity factors would corresspond to the followi ng el I i ptical angl es

$\begin{array}{cccc}\begin{array}{c}\text { el ement } \\ \text { number }\end{array} & \begin{array}{c}\text { thet a } \\ \text { (radi ans) }\end{array} & \begin{array}{c}\text { thet a } \\ \text { (degr ees) }\end{array} & \text { thet a/ ( pi / 2) } \\ 1 & 0.302 \mathrm{E}-01 & 0.173 \mathrm{E}+01 & 0.192 \mathrm{E}-01 \\ 2 & 0.242 \mathrm{E}+00 & 0.138 \mathrm{E}+02 & 0.154 \mathrm{E}+00 \\ 3 & 0.604 \mathrm{E}+00 & 0.346 \mathrm{E}+02 & 0.385 \mathrm{E}+00 \\ 4 & 0.967 \mathrm{E}+00 & 0.554 \mathrm{E}+02 & 0.615 \mathrm{E}+00 \\ 5 & 0.133 \mathrm{E}+01 & 0.762 \mathrm{E}+02 & 0.846 \mathrm{E}+00 \\ 6 & 0.154 \mathrm{E}+01 & 0.883 \mathrm{E}+02 & 0.981 \mathrm{E}+00\end{array}$

nozzl e corner mesh parameters(generated) :

case no. $(\mathrm{i} n d x 1)=2$ 
r1 $\quad=0.38000 \mathrm{E}+02$

$\begin{array}{lll}\text { ysht } & = & 0.10000 \mathrm{E}+03 \\ & = & 0.10000 \mathrm{E}+03\end{array}$

the computed stress intensity factors woul d corresspond to the following el I i ptical angl es

\begin{tabular}{|c|c|c|c|c|c|}
\hline $\begin{array}{l}\text { node } \\
\text { number }\end{array}$ & $\begin{array}{c}\text { thet a } \\
\text { (radi ans) }\end{array}$ & $\begin{array}{c}\text { thet a } \\
\text { ( degrees) }\end{array}$ & thet $\mathrm{a} /$ ( pi / & & \\
\hline $\begin{array}{r}1 \\
2 \\
3 \\
4 \\
5 \\
6 \\
7 \\
8 \\
9 \\
10 \\
11 \\
12 \\
13\end{array}$ & $\begin{array}{l}\text { 0. } 000 \mathrm{E}+00 \\
0.302 \mathrm{E}-01 \\
0.604 \mathrm{E}-01 \\
0.242 \mathrm{E}+00 \\
0.423 \mathrm{E}+00 \\
0.604 \mathrm{E}+00 \\
0.785 \mathrm{E}+00 \\
0.967 \mathrm{E}+00 \\
0.115 \mathrm{E}+01 \\
0.133 \mathrm{E}+01 \\
0.151 \mathrm{E}+01 \\
0.154 \mathrm{E}+01 \\
0.157 \mathrm{E}+01\end{array}$ & $\begin{array}{l}\text { 0. } 000 \mathrm{E}+00 \\
0.173 \mathrm{E}+01 \\
0.346 \mathrm{E}+01 \\
0.138 \mathrm{E}+02 \\
0.242 \mathrm{E}+02 \\
0.346 \mathrm{E}+02 \\
0.450 \mathrm{E}+02 \\
0.554 \mathrm{E}+02 \\
0.658 \mathrm{E}+02 \\
0.762 \mathrm{E}+02 \\
0.865 \mathrm{E}+02 \\
0.883 \mathrm{E}+02 \\
0.900 \mathrm{E}+02\end{array}$ & $\begin{array}{l}\text { 0. } 000 \mathrm{E}+00 \\
0.192 \mathrm{E}-01 \\
0.385 \mathrm{E}-01 \\
0.154 \mathrm{E}+00 \\
0.269 \mathrm{E}+00 \\
0.385 \mathrm{E}+00 \\
0.500 \mathrm{E}+00 \\
0.615 \mathrm{E}+00 \\
0.731 \mathrm{E}+00 \\
0.846 \mathrm{E}+00 \\
\text { 0. } 962 \mathrm{E}+00 \\
0.981 \mathrm{E}+00 \\
0.100 \mathrm{E}+01\end{array}$ & $\begin{array}{l}\text { 0. } 900 \mathrm{E}+02 \\
0.883 \mathrm{E}+02 \\
0.865 \mathrm{E}+02 \\
0.762 \mathrm{E}+02 \\
0.658 \mathrm{E}+02 \\
0.554 \mathrm{E}+02 \\
0.450 \mathrm{E}+02 \\
0.346 \mathrm{E}+02 \\
0.242 \mathrm{E}+02 \\
0.138 \mathrm{E}+02 \\
0.346 \mathrm{E}+01 \\
0.173 \mathrm{E}+01 \\
0.000 \mathrm{E}+00\end{array}$ & $\begin{array}{l}\text { 0. } 707 \mathrm{E}+00 \\
0.708 \mathrm{E}+00 \\
0.709 \mathrm{E}+00 \\
\text { 0. } 736 \mathrm{E}+00 \\
\text { 0. } 783 \mathrm{E}+00 \\
\text { 0. } 838 \mathrm{E}+00 \\
\text { 0. } 889 \mathrm{E}+00 \\
\text { 0. } 933 \mathrm{E}+00 \\
\text { 0. } 967 \mathrm{E}+00 \\
\text { 0. } 989 \mathrm{E}+00 \\
\text { 0. } 999 \mathrm{E}+00 \\
\text { 0. } 100 \mathrm{E}+01 \\
\text { 0. } 100 \mathrm{E}+01\end{array}$ \\
\hline
\end{tabular}

angul ar di vi si on - nozzl e

$\begin{array}{rr}\text { di v } & \text { angl e } \\ 1 & 0.00000 \\ 2 & 1.25689 \\ 3 & 2.51378 \\ 4 & 3.77067 \\ 5 & 5.02756 \\ 6 & 7.54134 \\ 7 & 10.05512 \\ 8 & 14.17148 \\ 9 & 18.28785 \\ 10 & 22.04923 \\ 11 & 25.81061 \\ 12 & 30.88520 \\ 13 & 35.95980 \\ 14 & 45.69245 \\ 15 & 55.42510 \\ 16 & 72.71255 \\ 17 & 90.00000\end{array}$

angul ar di vi si on - vessel

$\begin{array}{rr}\text { di v } & \text { angl e } \\ 1 & 0.83525 \\ 2 & 15.69604 \\ 3 & 30.55683 \\ 4 & 45.41762 \\ 5 & 60.27842 \\ 6 & 75.13921 \\ 7 & 90.00000\end{array}$

mesh generation parameters - subrouti ne revol v :

$\begin{array}{llr}\text { i n } & = & 3 \\ \text { v } & = & 3 \\ \text { x } & = & 5 \\ \text { y } & = & 9 \\ \text { cf } & = & 11 \\ \text { st } & = & 5 \\ \text { i ndx1 } & = & 2 \\ \text { nx } & = & 25 \\ \text { ny } & = & 13 \\ \text { nz1 } & = & 3 \\ \text { nz2 } & = & 3 \\ \text { nz3 } & = & 0 \\ \text { nozshp } & = & 2\end{array}$


perturbation vect or for crack front nodes

$\begin{array}{rrrr}\text { node } & \mathrm{d} 1 & \mathrm{~d} 2 & \mathrm{~d} 3 \\ 1 & 0.100000 \mathrm{E}+01 & 0.000000 \mathrm{E}+00 & 0.000000 \mathrm{E}+00 \\ 2 & 0.100000 \mathrm{E}+01 & 0.000000 \mathrm{E}+00 & 0.000000 \mathrm{E}+00 \\ 3 & 0.100000 \mathrm{E}+01 & 0.000000 \mathrm{E}+00 & 0.000000 \mathrm{E}+00 \\ 4 & 0.977101 \mathrm{E}+00 & 0.212778 \mathrm{E}+00 & 0.000000 \mathrm{E}+00 \\ 5 & 0.910891 \mathrm{E}+00 & 0.412647 \mathrm{E}+00 & 0.000000 \mathrm{E}+00 \\ 6 & 0.822262 \mathrm{E}+00 & 0.569109 \mathrm{E}+00 & 0.000000 \mathrm{E}+00 \\ 7 & 0.707107 \mathrm{E}+00 & 0.707107 \mathrm{E}+00 & 0.000000 \mathrm{E}+00 \\ 8 & 0.569106 \mathrm{E}+00 & 0.822264 \mathrm{E}+00 & 0.000000 \mathrm{E}+00 \\ 9 & 0.412650 \mathrm{E}+00 & 0.910890 \mathrm{E}+00 & 0.000000 \mathrm{E}+00 \\ 10 & 0.212774 \mathrm{E}+00 & 0.977101 \mathrm{E}+00 & 0.000000 \mathrm{E}+00 \\ 11 & 0.000000 \mathrm{E}+00 & 0.100000 \mathrm{E}+01 & 0.000000 \mathrm{E}+00 \\ 12 & 0.000000 \mathrm{E}+00 & 0.100000 \mathrm{E}+01 & 0.000000 \mathrm{E}+00 \\ 13 & 0.000000 \mathrm{E}+00 & 0.100000 \mathrm{E}+01 & 0.000000 \mathrm{E}+00\end{array}$

number of nodes i $\mathrm{n}$ adi na crack model. . . . . $($ nod $)=2616$

number of crack tip el ements. . . . . . $($ nel t $)=96$

first el ement on crack front. . . . . . . $($ nre2 $)=49$

first el ement of out er regi on ...... . $($ nre3 $)=0$

number of regul ar el ement s. . . . . . $\quad($ nel $)=408$

number of i nternal pres el ements... . . (nprel $)=218$

number of crack face pres el ements . . . . (nprcf $)=0$ 


\section{APPENDIX C MATHCAD DATA SHEETS WITH EVALUATION OF APPROXIMATE STRESS-INTENSITY FACTORS USING THE GUZHONG AND QICHAO (1990) APPROXIMATION}


Project: HFIR Vessel Life Extension

SHEET 1 OF 7

Report. No: ORNL/TM-2000/70

Calculation by: P. T. Williams, Ph.D., P.E. Date: 04 February 2000

\section{Reference}

1. C. Guozhong and H. Qichao, "Approximate Stress-Intensity Factor Solutions for Nozzle Corner Cracks," International Journal of Pressure Vessels and Piping 42, (1990) 75-96.

\section{Geometry Data (inches)}

$$
\begin{array}{ll}
a:=0.5 & d:=20.0 \\
r_{i}:=7.0 & D:=97.375 \\
t:=6.0 & \theta:=0,2 . .90 \\
T:=3.125 &
\end{array}
$$

\section{Vessel Hoop stress (ksi)}

$$
\sigma:=13.986
$$

\section{Stress Concentration Factor Model}

Decock's (1973) Equation for Elastic Concentration Factor J. Decock, "Determination of stress Concentration Factors and Fatigue Assessment of Flush and Extruded Nozzles in welded Pressure vessels," Conf. Pres. Ves. Techno7., Part II, (1973) 821-834.

$$
\begin{aligned}
& k_{e}:=\frac{\left[2.0+2.0 \cdot\left(\frac{d}{D}\right) \cdot \sqrt{\frac{(d \cdot t)}{(D \cdot T)}}+1.25 \cdot\left(\frac{d}{D}\right) \cdot\left(\frac{D}{T}\right)\right]}{1.0+\left(\frac{t}{T}\right) \cdot \sqrt{\frac{(d \cdot t)}{(D \cdot T)}}} \\
& k_{e}=4.651
\end{aligned}
$$

Stress Concentration Factor at $(r, \theta)$

$B:=2.7-2 . \sqrt{\frac{t}{d}}$

$B=1.605$

$$
k_{t}(x, y):=1 .+\left(k_{e}-1 \cdot\right) \cdot\left[\frac{1}{1+\frac{\pi \cdot x}{4.0 \cdot r_{i}} \cdot\left(\sin \left(y \cdot \frac{\pi}{180 .}\right)+\cos \left(y \cdot \frac{\pi}{180 .}\right)\right.}\right)^{B}
$$


Local Stress-Intensity Factor $(k s i \sqrt{\text { in }})$

$K_{I}(x, y):=\left[1.43-0.24 \cdot\left(\sin \left(y \cdot \frac{\pi}{180 .}\right)+\cos \left(y \cdot \frac{\pi}{180 .}\right)\right)\right] \cdot\left(\sigma \cdot k_{t}(x, y)\right) \cdot \frac{2 \cdot \sqrt{\pi x}}{\pi}$

Stress-Intensity Factor for $r_{f}=1.5$ in.

$$
a:=1.23
$$

\begin{tabular}{|c|c|c|c|}
\hline$\theta=$ & $k_{t}(a, \theta)=$ & $K_{I}(a, \theta)=$ & $k_{t}(a, \theta) \cdot \sigma=$ \\
\hline 0 & 3.967 & 82.620 & 55.479 \\
\hline 2 & 3.947 & 81.641 & 55.204 \\
\hline 4 & 3.928 & 80.708 & 54.941 \\
\hline 6 & 3.910 & 79.820 & 54.692 \\
\hline 8 & 3.894 & 78.978 & 54.455 \\
\hline 10 & \begin{tabular}{|l|}
3.878 \\
\end{tabular} & 78.180 & 54.231 \\
\hline 12 & 3.862 & 77.428 & 54.020 \\
\hline 14 & \begin{tabular}{|l}
3.848 \\
\end{tabular} & \begin{tabular}{|l|}
76.721 \\
\end{tabular} & 53.821 \\
\hline 16 & 3.835 & 76.058 & 53.636 \\
\hline 18 & \begin{tabular}{|l|}
3.823 \\
\end{tabular} & 75.440 & 53.462 \\
\hline 20 & 3.811 & 74.867 & 53.302 \\
\hline 22 & 3.800 & 74.339 & 53.154 \\
\hline 24 & \begin{tabular}{|l|}
3.791 \\
\end{tabular} & 73.855 & 53.018 \\
\hline 26 & 3.782 & 73.415 & 52.895 \\
\hline 28 & 3.774 & 73.020 & 52.784 \\
\hline 30 & 3.767 & 72.668 & 52.686 \\
\hline 32 & 3.761 & 72.361 & 52.600 \\
\hline 34 & 3.756 & 72.098 & 52.526 \\
\hline 36 & 3.751 & 71.879 & 52.465 \\
\hline 38 & \begin{tabular}{|l|}
3.748 \\
\end{tabular} & \begin{tabular}{|l|}
71.703 \\
\end{tabular} & 52.416 \\
\hline 40 & 3.745 & 71.572 & 52.379 \\
\hline 42 & \begin{tabular}{|l|}
3.743 \\
\end{tabular} & 71.484 & 52.355 \\
\hline 44 & \begin{tabular}{|l|}
3.742 \\
\end{tabular} & 71.440 & 52.343 \\
\hline 46 & 3.742 & 71.440 & 52.343 \\
\hline
\end{tabular}


SHEET 3 OF 7

Stress-Intensity Factor for $r_{f}=1.25$ in.

$a:=1.043$

\begin{tabular}{|c|c|c|c|}
\hline$\theta=$ & $k_{t}(a, \theta)=$ & $K_{I}(a, \theta)=$ & $k_{t}(a, \theta) \cdot \sigma=$ \\
\hline 0 & 4.057 & 77.805 & 56.737 \\
\hline 2 & 4.039 & \begin{tabular}{|l|}
76.933 \\
\end{tabular} & 56.491 \\
\hline 4 & 4.022 & \begin{tabular}{|l|}
76.100 \\
\end{tabular} & 56.257 \\
\hline 6 & 4.006 & 75.307 & 56.034 \\
\hline 8 & 3.991 & 74.553 & 55.823 \\
\hline 10 & 3.977 & \begin{tabular}{|l|}
73.839 \\
\end{tabular} & 55.622 \\
\hline 12 & 3.963 & 73.164 & 55.433 \\
\hline 14 & 3.951 & 72.530 & 55.255 \\
\hline 16 & 3.939 & 71.934 & 55.088 \\
\hline 18 & 3.928 & \begin{tabular}{|l|}
71.379 \\
\end{tabular} & 54.932 \\
\hline 20 & 3.917 & 70.863 & 54.787 \\
\hline 22 & 3.908 & 70.387 & 54.654 \\
\hline 24 & 3.899 & \begin{tabular}{|l|}
69.951 \\
\end{tabular} & 54.532 \\
\hline 26 & 3.891 & 69.554 & 54.421 \\
\hline 28 & 3.884 & 69.197 & 54.321 \\
\hline 30 & 3.878 & 68.880 & 54.232 \\
\hline 32 & 3.872 & 68.603 & 54.154 \\
\hline 34 & 3.867 & 68.365 & 54.088 \\
\hline 36 & 3.863 & 68.167 & 54.032 \\
\hline 38 & 3.860 & 68.008 & 53.988 \\
\hline 40 & 3.858 & \begin{tabular}{|l|}
67.889 \\
\end{tabular} & 53.955 \\
\hline 42 & 3.856 & 67.810 & 53.932 \\
\hline 44 & 3.855 & 67.770 & 53.921 \\
\hline \begin{tabular}{|l|}
46 \\
\end{tabular} & 3.855 & 67.770 & 53.921 \\
\hline
\end{tabular}


Stress-Intensity Factor for $r_{f}=0.75$ in.

$a:=0.647$

\begin{tabular}{|c|c|c|c|}
\hline$\theta=$ & $k_{t}(a, \theta$ & $K_{I}(a, \theta$ & $k_{t}(a$, \\
\hline 0 & 4.262 & 64.387 & 59.614 \\
\hline 2 & \begin{tabular}{|l|}
4.250 \\
\end{tabular} & \begin{tabular}{|l|}
63.760 \\
\end{tabular} & 59.444 \\
\hline 4 & 4.239 & 63.160 & 59.282 \\
\hline 6 & \begin{tabular}{|l}
4.228 \\
\end{tabular} & 62.586 & 59.127 \\
\hline 8 & \begin{tabular}{|l}
4.217 \\
\end{tabular} & \begin{tabular}{|l|}
62.039 \\
\end{tabular} & 58.979 \\
\hline 10 & 4.207 & 61.520 & 58.839 \\
\hline 12 & 4.198 & 61.028 & 58.707 \\
\hline 14 & \begin{tabular}{|l}
4.189 \\
\end{tabular} & 60.564 & 58.581 \\
\hline 16 & 4.180 & 60.128 & 58.464 \\
\hline 18 & 4.172 & \begin{tabular}{|l|}
59.721 \\
\end{tabular} & 58.354 \\
\hline 20 & 4.165 & 59.341 & 58.252 \\
\hline 22 & 4.158 & 58.991 & 58.157 \\
\hline 24 & 4.152 & 58.669 & 58.070 \\
\hline 26 & 4.146 & 58.376 & 57.991 \\
\hline 28 & \begin{tabular}{|l|}
4.141 \\
\end{tabular} & 58.112 & 57.920 \\
\hline 30 & 4.137 & 57.877 & 57.857 \\
\hline 32 & 4.133 & 57.671 & 57.801 \\
\hline 34 & 4.129 & 57.494 & 57.754 \\
\hline 36 & \begin{tabular}{|l}
4.127 \\
\end{tabular} & 57.347 & 57.714 \\
\hline 38 & 4.124 & 57.229 & 57.682 \\
\hline 40 & 4.123 & 57.141 & 57.658 \\
\hline 42 & \begin{tabular}{|l|}
4.121 \\
\end{tabular} & 57.082 & 57.643 \\
\hline \begin{tabular}{|l|}
44 \\
\end{tabular} & \begin{tabular}{|l}
4.121 \\
\end{tabular} & 57.052 & 57.635 \\
\hline 46 & \begin{tabular}{|l}
4.121 \\
\end{tabular} & 57.052 & 57.635 \\
\hline
\end{tabular}


Stress-Intensity Factors for $r_{f}=0.5$ in.

$a:=0.397$

\begin{tabular}{|c|c|c|c|}
\hline$\theta=$ & $k_{t}(a, \theta)=$ & $K_{I}(a, \theta)=$ & $k_{t}(a, \theta) \cdot \sigma=$ \\
\hline 0 & \begin{tabular}{|l|}
4.404 \\
\end{tabular} & 52.113 & 61.596 \\
\hline 2 & \begin{tabular}{|l|}
4.396 \\
\end{tabular} & 51.659 & 61.484 \\
\hline 4 & 4.388 & 51.223 & 61.377 \\
\hline 6 & 4.381 & 50.806 & 61.275 \\
\hline 8 & 4.374 & 50.408 & 61.177 \\
\hline 10 & \begin{tabular}{|l|}
4.367 \\
\end{tabular} & 50.029 & 61.084 \\
\hline 12 & \begin{tabular}{|l|}
4.361 \\
\end{tabular} & 49.669 & 60.996 \\
\hline 14 & \begin{tabular}{|l|}
4.355 \\
\end{tabular} & 49.329 & 60.912 \\
\hline 16 & \begin{tabular}{|l|}
4.350 \\
\end{tabular} & 49.010 & \begin{tabular}{|l|}
60.834 \\
\end{tabular} \\
\hline \begin{tabular}{|l|}
18 \\
\end{tabular} & \begin{tabular}{|l|}
4.344 \\
\end{tabular} & 48.710 & 60.760 \\
\hline 20 & \begin{tabular}{|l|}
4.339 \\
\end{tabular} & 48.431 & 60.692 \\
\hline 22 & 4.335 & 48.173 & 60.629 \\
\hline 24 & \begin{tabular}{|l|}
4.331 \\
\end{tabular} & 47.936 & 60.570 \\
\hline 26 & 4.327 & 47.719 & 60.517 \\
\hline 28 & 4.324 & 47.524 & 60.470 \\
\hline 30 & \begin{tabular}{|l|}
4.321 \\
\end{tabular} & 47.350 & 60.427 \\
\hline 32 & 4.318 & 47.198 & 60.390 \\
\hline 34 & \begin{tabular}{|l|}
4.316 \\
\end{tabular} & 47.067 & 60.358 \\
\hline 36 & 4.314 & 46.958 & 60.331 \\
\hline 38 & \begin{tabular}{|l|}
4.312 \\
\end{tabular} & 46.871 & 60.309 \\
\hline 40 & \begin{tabular}{|l|}
4.311 \\
\end{tabular} & 46.805 & 60.293 \\
\hline 42 & \begin{tabular}{|l|}
4.310 \\
\end{tabular} & 46.761 & 60.283 \\
\hline 44 & 4.310 & 46.740 & 60.277 \\
\hline 46 & 4.310 & 46.740 & 60.277 \\
\hline
\end{tabular}


Stress-Intensity Factor for $r_{f}=0.25$ in.

$a:=0.209$

\begin{tabular}{|c|c|c|c|}
\hline$\theta=$ & $k_{t}(a$, & $K_{I}(a$, & $k_{t}(a$, \\
\hline 0 & \begin{tabular}{|l|}
4.517 \\
\end{tabular} & 38.784 & 63.180 \\
\hline 2 & 4.513 & 38.478 & 63.118 \\
\hline 4 & 4.509 & 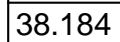 & 63.058 \\
\hline 6 & \begin{tabular}{|l|}
4.505 \\
\end{tabular} & 37.902 & \begin{tabular}{|l|}
63.001 \\
\end{tabular} \\
\hline 8 & \begin{tabular}{|l|}
4.501 \\
\end{tabular} & 37.632 & 62.947 \\
\hline 10 & \begin{tabular}{|l|}
4.497 \\
\end{tabular} & \begin{tabular}{|l|}
37.375 \\
\end{tabular} & \begin{tabular}{|l|}
62.895 \\
\end{tabular} \\
\hline 12 & \begin{tabular}{|l|}
4.493 \\
\end{tabular} & 37.131 & 62.845 \\
\hline 14 & 4.490 & 36.900 & 62.798 \\
\hline 16 & \begin{tabular}{|l|}
4.487 \\
\end{tabular} & 36.682 & \begin{tabular}{|l|}
62.754 \\
\end{tabular} \\
\hline 18 & \begin{tabular}{|l|}
4.484 \\
\end{tabular} & 36.478 & 62.713 \\
\hline 20 & 4.481 & 36.288 & 62.675 \\
\hline 22 & 4.479 & 36.112 & 62.639 \\
\hline \begin{tabular}{|l|}
24 \\
\end{tabular} & \begin{tabular}{|l|}
4.476 \\
\end{tabular} & 35.949 & 62.606 \\
\hline 26 & \begin{tabular}{|l|}
4.474 \\
\end{tabular} & 35.801 & 62.576 \\
\hline 28 & 4.472 & 35.668 & 62.549 \\
\hline 30 & \begin{tabular}{|l|}
4.471 \\
\end{tabular} & 35.549 & 62.525 \\
\hline \begin{tabular}{|l|}
32 \\
\end{tabular} & \begin{tabular}{|l|}
4.469 \\
\end{tabular} & 35.444 & 62.504 \\
\hline 34 & 4.468 & 35.355 & 62.486 \\
\hline 36 & \begin{tabular}{|l|}
4.467 \\
\end{tabular} & 35.280 & 62.471 \\
\hline 38 & 4.466 & 35.220 & 62.458 \\
\hline 40 & \begin{tabular}{|l|}
4.465 \\
\end{tabular} & 35.175 & 62.449 \\
\hline \begin{tabular}{|l|}
42 \\
\end{tabular} & \begin{tabular}{|l|}
4.465 \\
\end{tabular} & 35.145 & 62.443 \\
\hline \begin{tabular}{|l|}
44 \\
\end{tabular} & \begin{tabular}{|l|}
4.464 \\
\end{tabular} & 35.130 & 62.440 \\
\hline \begin{tabular}{|l|}
46 \\
\end{tabular} & \begin{tabular}{|l|}
4.464 \\
\end{tabular} & 35.130 & 62.440 \\
\hline
\end{tabular}


$\underline{\text { Nomenclature }}$

$a$ = flaw depth

$R_{i}=$ vessel inner radius

$r_{i}=$ nozzle inner radius

$t=$ nozzle wall thickness

$T=$ vessel wall thickness

$d=$ mean nozzle diameter

$D=$ mean vessel diameter

$\sigma=$ vessel hoop stress

$\theta=$ flaw front angle

$k_{e}=$ elastic concentration factor

$k_{t}=$ local stress concentration factor

$B=$ exponent for $k_{t}$

$K_{I}=$ local applied stress intensity factor 


\section{INTERNAL DISTRIBUTION}

1. B. R. Bass

2. S. E. Burnette

3. R. D. Cheverton

4. S. J. Chang

5. K. W. Childs

6. D. H. Cook

7. T. L. Dickson

8. D. M. Hetrick

9-11. J. R. Inger

12. S. K. Iskander

13. M. A. Kuliasha
14. W. J. McAfee

15. L. D. Proctor

16. D. L. Selby

17-20. P. T. Williams

21. Research Reactors Division - DCC $\mathrm{RC}$

22. ORNL Patent Office

23-24. Central Research Library

25-26. Laboratory Records (for OSTI)

27. Laboratory Records, RC 